\title{
Ejes claves del modelo educativo socioformativo para la formación universitaria en el marco de la transformación hacia el desarrollo social sostenible
}

\author{
Jorge E. Martínez-Iñiguez', Sergio Tobón ${ }^{2 *}$ y Jesús A. Soto-Curiel1 \\ (1) Facultad de Ciencias Humanas, Universidad Autónoma de Baja California, Blvd. Castellón y Lombardo Toledano \\ S/N, colonia Conjunto Urbano Esperanza, Mexicali, Baja California, C.P. 21350, México. \\ (correo-e: jorge.martinez43@uabc.edu.mx; adolfo.soto@uabc.edu.mx). \\ (2) Centro Universitario CIFE, Calle Tabachín 514, colonia Bellavista, Cuernavaca, Morelos, C.P. 62140, México. \\ (correo-e: stobon5@gmail.com).
}

* Autor a quien debe ser dirigida la correspondencia.

Recibido May. 11, 2020; Aceptado Jul. 7, 2020; Versión final Sep. 1, 2020, Publicado Feb. 2021

\begin{abstract}
Resumen
El objetivo del presente estudio fue valorar desde el enfoque educativo de la socioformación, las características de un modelo educativo para formar en el marco de los retos del desarrollo social sostenible. Se realizó un análisis documental por medio de las ocho categorías de la cartografía conceptual. Los resultados indican que el modelo educativo socioformativo orienta la formación integral para que se implementen proyectos que contribuyan a lograr el desarrollo social sostenible, con base en la colaboración, la transversalidad y la vinculación con las comunidades. Implica autoevaluar en todos los escenarios su ejecución e implementar mejoras continuas con el apoyo de todos para que no se quede en un simple discurso, tal y como ha ocurrido en muchas universidades. En conclusión, el modelo educativo socioformativo es una alternativa de cambio a las actuales prácticas educativas que poco o nulo impacto tienen en la formación universitaria para el desarrollo social sostenible.
\end{abstract}

Palabras clave: desarrollo social sostenible; educación superior; modelo educativo; socioformación

\section{Key axes of the socioformative educational model for university training in the transformation framework towards sustainable social development}

\begin{abstract}
The objective of the present study was to assess the characteristics of a training educational model within the framework of sustainable social development challenges by applying a socioformation educational approach. Using the eight categories of conceptual cartography, a documentary analysis was performed. The results showed that the socioformative educational model guides integral training. This serves to implement projects that contribute to achieving sustainable social development based on collaboration, mainstreaming, and linking with communities. This also implies assessing its execution in all possible scenarios and implementing continuous improvements with widespread support by all sectors, so that the socioformative educational model does not remain in a simple speech as has happened at multiple universities. In conclusion, the socioformative educational model is an alternative to modify current educational practices that have little or no impact on training in sustainable social development in higherlevel education.
\end{abstract}




\section{INTRODUCCIÓN}

La sociedad en la que vivimos se encuentra en un proceso de constantes y profundos cambios sociales, políticos y económicos, por lo que la educación y el conocimiento son elementos que se consideran importantes para afrontar los retos y desafíos expuestos en la actualidad, así como aquellos que están por venir a lo largo del siglo XXI (Echeverría y Martínez, 2018; Heyneman y Lee, 2016) como consecuencia de la globalización y la sociedad del conocimiento (Ricaurte, 2016; Tünnermann, 2008), además de la emergente industria 4.0 o cuarta revolución industrial (llori y Ajagunna, 2020; Schwab, 2016). Cabe señalar que el panorama actual comenzó a gestarse desde la década de 1960 a causa de que las diversas agencias bilaterales y multilaterales como la Ayuda Oficial al Desarrollo (AOD), la Organización para la Cooperación y el Desarrollo Económicos (OCDE), la UNESCO (por sus siglas en inglés), el Banco Mundial, las Naciones Unidas, entre otras, con el paso del tiempo han venido emitiendo recomendaciones que buscan persuadir a los gobiernos con la intención de que sus políticas promuevan la transformación de sus sistemas e instituciones educativas, y así poder contribuir al desarrollo socioeconómico de sus países (Heynema y Lee, 2016). Ante este contexto, se considera importante que las personas formadas en el nivel superior desarrollen nuevas competencias para ámbitos profesionales que todavía no existen o apenas están iniciando en un determinado contexto (Echeverría y Martínez, 2018).

Por otra parte, más que los cambios descritos en materia socioeconómica, el principal reto para las comunidades es transformarse para formar ciudadanos integrales que contribuyan al desarrollo social sostenible, concepto en el cual confluyen una serie de metas articuladas, tales como la mejora de la calidad de vida, el acceso a servicios de salud de calidad, el desarrollo de las empresas, el fortalecimiento de la economía, la generación de empleos, la mejora de la educación, la convivencia, la inclusión, el cuidado del ambiente y la protección de la biodiversidad, entre otros ejes, con base en el trabajo colaborativo de diferentes actores (García-Valdés y Juárez-Hernández, 2019; Jim y Shen, 2016; United Nations, 2015; UNESCO, 2017; Tobón y Luna-Nemecio, 2020).

De acuerdo con diversas investigaciones, dentro del desarrollo sostenible se integran los componentes social (que tiene que ver con la familia, la educación, las generaciones futuras, etc.), económico (relacionado con el comercio, las finanzas, entre otros aspectos) y ambiental (que toma en cuenta la preservación de los recurso naturales, etc.) en la edificación de un futuro que sea capaz de satisfacer las necesidades de las nuevas generaciones (Andriamihaja et al., 2021; Juárez-Hernández et al., 2019); empero, de acuerdo con Juárez-Hernández et al., (2019) la realidad es que el componente social ha sido el menos explorado en la construcción de una sociedad más justa y equitativa, aun cuando la participación social de diversos actores es esencial en la promoción de acciones que traten de erradicar los problemas que más aquejan a la humanidad. En este sentido, la educación con calidad es un eje de acción para transitar hacia la sostenibilidad, es decir, de establecer un proceso de formación de personas con visión holística y comprometidas con el progreso humano de la sociedad (Aliaga-Pacora y Luna-Nemecio, 2020; Colombo y Alves, 2017; Juárez-Hernández et al., 2019; United Nations, 2015). Por ello la importancia de que en la actualidad se hable de desarrollo social sostenible como un concepto de equilibrio de los componentes social, económico y ambiental.

Ahora bien, considerando que la formación para el desarrollo social sostenible es una característica a incluir dentro de los modelos educativos universitarios, es indispensable que las IES transiten de la enseñanza tradicional en las aulas a una formación con vinculación a las problemáticas reales que se presentan en los distintos escenarios sociales, organizacionales y comunitarios (Juárez-Hernández et. al., 2019), con la finalidad de posibilitar en las personas el desarrollo de profesionales con competencias en pensamiento crítico, aprendizaje activo y continuo, resolución de problemas complejos, gestión del conocimiento, trabajo colaborativo, inteligencia emocional, flexibilidad cognitiva, creatividad, transdisciplinariedad, emprendimiento creativo, interculturalidad, comunicación oral y escrita, comunicación oral y escrita en una segunda lengua, utilización de nuevas tecnologías, gestión de la calidad, entre otras competencias requeridas para afrontar los problemas del presente y futuro de la sociedad (Cebrián et al., 2020; Echeverría y Martínez, 2018; llori y Ajagunna, 2020; Tobón, 2017; OECD, 2017; Schawb, 2016).

Si bien es cierto que los recientes modelos educativos establecidos por diversas IES han promovido un papel activo del alumno en la construcción de su propio aprendizaje, con base en el enfoque de competencias u otras corrientes educativas que otorgan un papel de guía o asesor al profesorado dentro de los procesos que conllevan a la formación integral del estudiante (Barbosa y Amariles, 2019; Gaffigna et al., 2020; Martínez et al., 2017; Solís et al., 2015), la realidad es que las prácticas educativas que siguen ejerciendo las instituciones educativas todavía no logran contribuir de una manera sustancial a la mejora de la calidad de vida, ni a la solución de problemas que aquejan a la humanidad, debido a que estas mantienen en su esencia prácticas tradicionales dentro de su currículo, tales como: la evaluación de los aprendizajes a través de pruebas estandarizadas, la enseñanza de contenidos temáticos, el trabajo académico por 
asignaturas aisladas, entre otras (Martínez et al., 2019; Solís et al., 2015). En la mayoría de los casos el enfoque de competencias se ha quedado en el discurso (Glaesser, 2019; Guzmán, 2017; Martínez et al., 2017), o en procesos muy complejos de diseño curricular y microcurricular que pocos profesores comprenden (Díaz Barriga 2019). Además, el planteamiento de las competencias en diversas experiencias se asume desde enfoques que fueron planteados para otros momentos históricos (como el técnico-laboral, el constructivista o el socioconstructivista) (Díaz Barriga, 2019), pero no para el actual, que requiere procesos de transformación cultural, social, organizacional y política.

Por lo anterior, es necesario pasar del enfoque de competencias, hegemónico en la actualidad en las políticas para la educación superior (Díaz Barriga, 2019), a nuevas perspectivas que orienten la formación de ciudadanos emprendedores. En este sentido, se propone la socioformación, un enfoque de origen Latinoamericano que se viene construyendo desde principios del siglo XXI de manera colaborativa con las contribuciones de diversos investigadores, cuyo propósito es formar para lograr el desarrollo social sostenible, mediante el emprendimiento de proyectos articulados basados en la docencia, la investigación y la vinculación con la comunidad, las organizaciones y los problemas reales de la sociedad (Ambrosio, 2018; Martínez et al., 2017; Aguilar-Esteva et al., 2019). En esta propuesta, la finalidad es la transformación de las comunidades para que sean autogestoras del desarrollo, y esto implica el desarrollo de competencias (o capacidades o habilidades), pero también de otras acciones articuladas, mismas que requieren del trabajo colaborativo de todos los actores que interactúan en una comunidad educativa, del establecimiento de proyectos para la atención de situaciones y problemas reales, la gestión y co-creación del conocimiento con el apoyo de las nuevas tecnologías, los procesos de mejora continua del talento humano de las personas, entre otras (Ambrosio, 2018).

En la actualidad, se cuenta con aportaciones del enfoque de la socioformación en diversos campos, tales como: la gestión curricular (Martínez et al., 2019; Tobón, 2017), la cultura de calidad en educación superior (Martínez et al., 2017), la función directiva en instituciones educativas (Tobón et al., 2020), el talento humano (Tobón y Luna-Nemecio, 2020), la evaluación de modelos educativos (Aguilar-Esteva et al., 2019), la formación para la sostenibilidad (Aliaga-Pacora y Luna-Nemecio, 2020; García-Valdés y JuárezHernández, 2019; Juárez-Hernández et al., 2019), entre otros.

De acuerdo con el el presente contexto, es necesario determinar los ejes esenciales que debe considerar el modelo educativo de una universidad desde propuestas educativas que tomen en cuenta las características formativas que se requieren para afrontar los retos de la sociedad actual. Es por ello que se ha realizado un estudio conceptual en torno al modelo educativo para las universidades desde la socioformación, con los siguientes propósitos: 1) describir la noción de modelo educativo acorde con los retos del desarrollo social sostenible; 2) identificar las características que debe poseer un modelo educativo desde la socioformación; 3) proponer un proceso metodológico para construir un modelo educativo universitario que responda a los retos y necesidades de la comunidad; y 4) situar el desarrollo de futuros estudios que posibiliten el establecimiento de nuevos modelos educativos pertinentes a las características de las comunidades educativas del nivel superior desde la socioformación.

\section{METODOLOGÍA}

Se describe el tipo de estudio efectuado con su respectiva estrategia de investigación, así como también se presentan los criterios de selección de documentos utilizados.

\section{Tipo de Estudio}

En congruencia con los propósitos del estudio, se realizó un análisis documental, el cual consiste en un proceso que posibilita al investigador la búsqueda, organización y representación del conocimiento obtenido de diversas fuentes, con la finalidad de generar un documento con nuevo conocimiento en relación con un tema o problema de estudio (Peña y Pirela, 2007). Como estrategia de investigación se recurrió a la cartografía conceptual, la cual fue adaptada por Tobón en el marco del enfoque socioformativo (Tobón, 2013) con la finalidad de estudiar un tema, concepto, teoría, metodología o problema a través de ocho categorías de análisis que se muestran en la Tabla 1, mismas que facilitan la sistematización de la información disponible, la valoración de los avances científicos, la determinación de posibles vacíos conceptuales, así como situar la realización de nuevas investigaciones (Ortega et al., 2015). Dicha estrategia es pertinente cuando se busca aclarar o relacionar un tema con un enfoque o teoría, como es el caso de la presente investigación. De esta forma, se efectúo el estudio de varios documentos con base en algunas preguntas orientadoras establecidas en cada categoría de la cartografía conceptual, mismas que permitieron determinar los ejes claves del modelo educativo socioformativo en el marco del desarrollo social sostenible. 
Tabla 1: Categorías de análisis del estudio

\begin{tabular}{|l|l|}
\hline Categorías & Preguntas guía \\
\hline $\begin{array}{l}\text { Noción del modelo educativo } \\
\text { socioformativo }\end{array}$ & $\begin{array}{l}\text { ¿Qué es un modelo educativo socioformativo para el desarrollo social } \\
\text { sostenible? }\end{array}$ \\
\hline $\begin{array}{l}\text { Categorización del modelo educativo } \\
\text { desde la socioformación }\end{array}$ & $\begin{array}{l}\text { ¿Qué es formar para el desarrollo social sostenible desde el modelo } \\
\text { educativo socioformativo? }\end{array}$ \\
\hline $\begin{array}{l}\text { Características del modelo educativo } \\
\text { socioformativo para el desarrollo social } \\
\text { sostenible }\end{array}$ & $\begin{array}{l}\text { ¿Cuáles son las características que debe tener el modelo educativo } \\
\text { socioformativo articulado al desarrollo social sostenible? }\end{array}$ \\
\hline $\begin{array}{l}\text { Diferenciación del modelo educativo } \\
\text { socioformativo }\end{array}$ & $\begin{array}{l}\text { ¿De qué otros conceptos cercanos se diferencia el modelo educativo } \\
\text { socioformativo orientado al desarrollo social sostenible? }\end{array}$ \\
\hline $\begin{array}{l}\text { División del modelo educativo } \\
\text { socioformativo }\end{array}$ & $\begin{array}{l}\text { ¿Cuáles son los ejes clave en los cuales se divide el modelo educativo } \\
\text { socioformativo para el desarrollo social sostenible? }\end{array}$ \\
\hline $\begin{array}{l}\text { Vinculación del modelo educativo } \\
\text { socioformativo }\end{array}$ & $\begin{array}{l}\text { ¿Con qué otros conceptos se relaciona el modelo educativo } \\
\text { socioformativo centrado en el desarrollo social sostenible? }\end{array}$ \\
\hline $\begin{array}{l}\text { Metodología de construcción del modelo } \\
\text { educativo socioformativo }\end{array}$ & $\begin{array}{l}\text { ¿Cuáles son los pasos esenciales para construir el modelo educativo } \\
\text { socioformativo enfocado en el desarrollo social sostenible? }\end{array}$ \\
\hline $\begin{array}{l}\text { Ejemplificación del modelo educativo } \\
\text { socioformativo: proceso de construcción }\end{array}$ & $\begin{array}{l}\text { ¿Cuál podría ser un ejemplo pertinente de elaboración del modelo } \\
\text { educativo socioformativo para el desarrollo social sostenible? }\end{array}$ \\
\hline
\end{tabular}

\section{Criterios de selección de documentos}

Acorde con las metas del estudio, el análisis documental se basó en los siguientes criterios: 1) búsqueda de artículos, libros, capítulos de libro y documentos de trabajo publicados en español e inglés, provenientes de bases de datos como Scopus, Scielo, Web of Science y Google Académico, por lo que se utilizaron palabras clave como: modelo educativo, socioformación, educación superior, desarrollo sostenible, sostenibilidad y desarrollo social sostenible; 2 ) en cuanto a libros y documentos de trabajo, estos tenían que poseer autor, título, fecha de publicación y editorial; 3) la información consultada debía contribuir al desarrollo de alguna de las ocho categorías de análisis establecidas en la cartografía conceptual y comprender el periodo 2015-2020, empero, también se consultaron otros documentos de mayor antigüedad de acuerdo con las aportaciones brindadas al tema de investigación; y 4) de 60 documentos analizados, se seleccionaron 25 artículos, 6 libros, 1 capítulo de libro y 2 documentos de trabajo por considerar de relevancia su contribución al tema de investigación.

\section{RESULTADOS}

A continuación se presentan los resultados del estudio siguiendo los ocho ejes propuestos por la cartografía conceptual.

\section{Noción del modelo educativo socioformativo}

En la educación superior, el modelo educativo es un tema que se comienza a trabajar con profundidad. De acuerdo con Fresán et al. (2017), los modelos se empiezan a abordar desde el 2006 como respuesta a la pérdida de identidad que se ha venido presentando en las instituciones del nivel superior como consecuencia del seguimiento y cumplimiento de políticas evaluativas que, si bien han permitido mejorar los indicadores institucionales, estas han venido desvirtuando el quehacer educativo de las IES.

Por su parte, Tünnermann (2008) conceptualiza al modelo educativo como la concreción, en términos pedagógicos, de los paradigmas educativos que una institución educativa profesa, mismos que desde un punto de vista psicopedagógico toman como base los principios más vigentes de la teoría psicológica y pedagógica, por lo que en la actualidad un gran número de IES han recurrido a las teorías del constructivismo y del humanismo.

Considerando lo anterior, y tomando en cuenta los retos de la sociedad actual, el modelo educativo desde la socioformación debe ser una visión compartida de todos los actores de las IES en torno a la formación integral de profesionales que pongan en funcionamiento proyectos y acciones con impacto en lograr el desarrollo social sostenible (Martínez et al., 2019; Valles-Baca et al., 2019), en el marco de la sociedad del conocimiento, con inclusión, interculturalidad, integralidad, transversalidad, equidad y compromiso con la excelencia, a través de la articulación de la docencia, la investigación, la vinculación con las comunidades y la gestión, de tal manera que se puedan brindar soluciones específicas a problemas como la pobreza, la 
violencia, el narcotráfico, las adicciones, los embarazos no planeados, la contaminación del ambiente, etc. (Aguilar-Esteva et al., 2019; Martínez et al., 2019). El modelo educativo socioformativo, como visión compartida en su conjunto, implica que todos los actores contribuyan en su construcción, validación, ejecución y mejora, para asegurar que efectivamente tenga impacto en la formación necesaria para ayudar a la comunidad en su desarrollo integral (Aguilar-Esteva et al., 2019).

\section{Categorización del modelo educativo desde la socioformación}

A pesar de los constantes y acelerados cambios que presenta la sociedad del siglo XXI, las prácticas educativas en la mayoría de las universidades siguen estando centradas en formar para la sociedad industrial, porque tienen como principal meta formar en contenidos, trabajar por asignaturas, calificar con notas y tener una cultura industrial, en donde predomina la enseñanza en masas, el individualismo, el trabajo enciclopédico y poca o nula vinculación con las dificultades existentes en la sociedad (Martínez et al., 2019). Aunque diversas universidades integraron en su modelo educativo el enfoque de competencias y los cuatro aprendizajes clave de Delors (1996) (aprender a conocer, aprender a hacer, aprender a ser y aprender a convivir), esto casi no se aborda en la práctica (Guzmán, 2017; Martínez et al., 2017). Es por ello urgente el cambio en la cultura universitaria, de tal manera que la formación sea para el desarrollo social sostenible (Bauer et al., 2020).

¿Qué es el desarrollo social sostenible? Es trabajar de manera colaborativa entre todos los actores sociales por la mejora de la calidad de vida, la promoción de la salud y la prevención de las enfermedades, el desarrollo económico, la creación y el fortalecimiento de las empresas, la inclusión, la equidad, la convivencia, el fortalecimiento de la ciencia y la tecnología, la promoción de la cultura, el cuidado del ambiente y la protección de la biodiversidad, entre otros elementos, con base en proyectos que tengan continuidad, el trabajo en red, el uso progresivo de las energías limpias, la agricultura ecológica y la política basada en la honestidad y el compromiso con el bien común (United Nations, 2015; UNESCO, 2017). Una universidad que implemente el modelo educativo socioformativo, considera el desarrollo social sostenible y se observa en el abordaje de problemas del mundo real que benefician a la sociedad, a través de la colaboración, la gestión del conocimiento y la metodología de proyectos (Aguilar-Esteva et al., 2019; GarcíaRubio et al., 2019; Martínez et al., 2019), articulando las funciones básicas universitarias en torno a este propósito, tales como la docencia, la investigación, la extensión, la vinculación y los servicios (Jim y Shen, 2016; Tünnermann, 2008).

\section{Características del modelo educativo socioformativo para el desarrollo social sostenible}

Tradicionalmente, los modelos educativos implantados en las IES se han caracterizado por: 1) prácticas educativas orientadas a la apropiación de contenidos, pero sin aplicación en problemas del contexto; 2) modelo de formación industrial basado en la gestión del docente de todo el proceso, con base en asignaturas fragmentadas; 3) logro de evidencias con poco valor o pertinencia en el entorno; y 4) una evaluación que se ejecuta para calificar, no para formar (Díaz Barriga, 2019; Martínez et al., 2019; Solís et al., 2015). Contrario a lo anterior, el modelo educativo socioformativo forma para el desarrollo social sostenible, ya que posee como mínimo las siguientes características: resolución de problemas de alto impacto en el contexto, obtención de productos con valor en el entorno, evaluación orientada a la formación y mejora continua del desempeño, flexibilidad curricular, trabajo colaborativo de todos los actores educativos, proyecto ético de vida y pensamiento complejo (Martínez et al., 2019).

\section{Resolución de problemas de alto impacto en el contexto}

Es urgente que la formación de las universidades deje de ser enciclopédica y centrada en aprender contenidos, y en su lugar se oriente a lograr retos que ayuden al desarrollo social sostenible de las comunidades, a través de la búsqueda, comprensión, análisis, crítica, creación y aplicación del saber científico, tecnológico, comunitario y/o artístico, según sea el caso (Cebrián et al., 2020). Esto implica que la formación sea con base en la resolución de problemas desde el inicio, en todos los espacios microcurriculares (Ambrosio, 2018; Valles-Baca et al., 2019).

\section{Obtención de productos con valor en el entorno}

En la mayoría de las universidades las evidencias que deben presentar los alumnos se refieren a exámenes escritos, trabajos de revisión de temas y ejercicios. Únicamente se presentan algunos trabajos prácticos durante las prácticas o materias altamente prácticas. Esto hace que no se tenga impacto en el desarrollo de la comunidad y que los alumnos se formen aislados del contexto social y económico. Formar para el desarrollo social sostenible, tal y como lo propone la socioformación, implica trabajar con base en evidencias y productos pertinentes al contexto, más allá de la perspectiva academicista que ha predominado en las IES (Aguilar-Esteva et al., 2019; García-Valdés y Juárez-Hernández, 2019). 


\section{Evaluación orientada a la formación y a la resolución de problemas del contexto}

La evaluación, en una perspectiva socioformativa y considerando el desarrollo social sostenible, debe propender por la formación continua para que los alumnos identifiquen sus áreas de oportunidad y avancen en el desarrollo de su talento (Tobón y Luna-Nemecio, 2020). Además, debe servir para la resolución de problemas respecto a la mejora de las condiciones de vida, el progreso económico y el cuidado del ambiente (Jim y Shen, 2016). La parte de las calificaciones sólo debe ser una actividad de apoyo al desarrollo de las habilidades necesarias para afrontar el mundo real, y no el centro de la evaluación en una universidad (Díaz Barriga, 2019).

\section{Flexibilidad curricular}

Formar para el desarrollo social sostenible requiere que el currículo sea sencillo y posibilite la flexibilidad necesaria para que los estudiantes sean formados para afrontar los retos de alto impacto del contexto (UNESCO, 2017), por medio de un perfil de egreso que describa las características que debe poseer el profesional, tanto de forma general como ciudadano líder, con respecto al ámbito profesional que espera ejercer, brindando la posibilidad de varios itinerarios en función de sus intereses y talento (Tobón, 2017). La estructura curricular debe adoptar nuevas opciones, más allá de las asignaturas, como los proyectos formativos (Martínez et al., 2019), que se orientan a formar con base en la resolución de problemas. Además, un currículo flexible debe poder incorporar cambios y mejoras de manera rápida y ágil en función de los retos sociales y el trabajo coordinado de los diversos actores que participan (Jim y Shen, 2016; Martínez et al., 2019; Valles-Baca et al., 2019). En la sociedad del siglo XXI, el desarrollo del currículo debe ser una tarea permanente, involucrando a todos los actores educativos, y también a las organizaciones y la sociedad, de tal forma que ayude a afrontar los retos que se tienen en el entorno (llori y Ajagunna, 2020; Jim y Shen, 2016; Martínez et al., 2019).

\section{Trabajo colaborativo de todos los actores educativos}

El trabajo colaborativo es esencial para obtener soluciones con continuidad e impacto en los problemas que afectan el desarrollo social sostenible (Jim y Shen, 2016; United Nations, 2015; UNESCO, 2017). Implica que las personas trabajen de manera articulada en la resolución de problemas del contexto y logren productos con pertinencia, mediante un plan de acción compartido, la responsabilidad individual, el apoyo entre todos para lograr la formación necesaria, la comunicación asertiva, la distribución de funciones o tareas y la metacognición para mejorar lo que se hace de manera continua (Ambrosio, 2018). No es lo mismo que el trabajo en equipo o el trabajo en grupo porque estos aspectos no implican la articulación de las acciones de los diversos participantes.

\section{Proyecto ético de vida}

El proyecto ético de vida se refiere a actuar con responsabilidad, respeto, honestidad y equidad para contribuir al desarrollo de la comunidad, cuidar el ambiente y buscar la realización como ser humano, con un sentido claro de la vida, trabajando por el logro de metas que tenga impacto en mejorar las condiciones de vida a corto, mediano y largo plazo (Tobón y Luna-Nemecio, 2020). Esto requiere la apropiación y aplicación articulada del saber ser, conocer, convivir y hacer en el abordaje del conjunto de problemas más apremiantes de la sociedad (Ambrosio, 2018).

\section{Mejora continua}

La implementación del modelo educativo socioformativo en las IES se basa en la cultura de la mejora continua en todo lo que se hace (Aguilar-Esteva et al., 2019; Martínez et al., 2019). Para lograrlo, las IES deben autoevaluar y diagnosticar sus prácticas educativas por medio de la observación y el seguimiento a los problemas que abordan los alumnos y los productos que presentan en su formación, considerando los retos del desarrollo sostenible de cada comunidad (García-Valdés y Juárez-Hernández, 2019). También deben llevarse diagnósticos continuos del trabajo investigativo, de la vinculación con la comunidad y de la gestión, para identificar áreas de oportunidad y resolverlas, con base en la metacognición y la práctica de la humildad (Aguilar-Esteva et al., 2019; García-Valdés y Juárez-Hernández, 2019).

\section{Pensamiento complejo}

El pensamiento complejo integra los diferentes tipos de pensamiento y se refiere a la resolución de problemas con base en el análisis crítico, el pensamiento sistémico, la construcción del saber, la creatividad y la metacognición. En las IES, más que citar a Morin (1999), lo que debe hacerse es formar a los alumnos y docentes en las habilidades de pensamiento complejo. Además, se debe adoptar una cultura de trabajo transversal en la cual se articulen distintos campos y disciplinas para lograr soluciones de alto impacto a los problemas, mediante procesos curriculares incluyentes y articulados (Jim y Shen, 2016; Valles-Baca et al., 2019). 


\section{Diferenciación del modelo educativo socioformativo de otros modelos}

Un modelo educativo para el desarrollo social sostenible basado en la socioformación difiere de otros tipos de modelos educativos que actualmente aplican diversas universidades, como: el modelo academicista, el modelo por competencias y el modelo orientado a la sociedad del conocimiento. A continuación, se explican las principales diferencias:

\section{Modelo educativo academicista o con orientación disciplinar}

Le da una gran importancia a la apropiación de los contenidos, teorías y metodologías de las disciplinas como valor en sí mismo, sin buscar la aplicación en la resolución de problemas, o por lo menos esto solamente aparece al final de los procesos de formación (Eryaman, 2010). Se basa en asignaturas, y es común que un semestre posea muchas asignaturas con el fin de abordar todas las áreas de la disciplina o profesión, porque se asume que el estudiante debe tener un conocimiento esencial de todo. Aunque este modelo busca la apropiación del saber científico, prepara poco para afrontar los retos del desarrollo social sostenible. En este tipo de modelo se privilegian las exposiciones magistrales del profesor, la enseñanza pasiva de contenidos y la evaluación de tipo cuantitativa (Rolón, 2016).

\section{Modelo educativo basado en competencias}

Se adoptó hace más de una década en diversas políticas para la educación superior, siendo el enfoque que predomina en la actualidad en muchas IES (por lo menos en el discurso y el currículo), buscando que los estudiantes desarrollen un conjunto bien definido de competencias genéricas y específicas con base en la implementación de productos de aplicación en los programas universitarios (Barbosa y Amariles, 2019; Díaz Barriga, 2019; Gaffigna et al., 2020). Aunque el modelo ideal de las competencias plantea una formación teórica-práctica centrada en abordar tareas del contexto con articulación de las habilidades, conocimientos y actitudes, en la práctica esto ha sido muy difícil de ejecutar por factores relacionados con la falta de comprensión y asimilación por parte de los actores que interactúan dentro de las comunidades educativas, la carencia de fundamentos psicopedagógicos que explique sus implicaciones en el ámbito educativo (Guzmán, 2017), así como la falta de transformación de otros ejes del trabajo universitario, como el ámbito de la organización escolar, la cultura de calidad institucional y de los procesos de formación profesional (Díaz Barriga, 2019; Guzmán, 2017; Martínez et al., 2017). Además, el aprender a hacer o la realización de tareas tiene muchas interpretaciones que no implican transformar la formación ni tampoco abordar los retos del desarrollo social sostenible.

\section{Modelo educativo centrado en formar para la sociedad del conocimiento}

Es un modelo orientado a formar profesionales que puedan trabajar en el marco de la cultura digital, con base en el trabajo en equipo y la conformación de comunidades a partir de las redes sociales y otras aplicaciones, donde es muy importante aprender a buscar, organizar, crear y aplicar el conocimiento (Kangas y Aarrevaara, 2020; Ricaurte, 2016). El problema es que esto no implica ejecutar proyectos que lleven a la mejora de las condiciones de vida, al desarrollo económico y al cuidado del ambiente. De igual forma, las condiciones de desigualdad socioeconómica existentes en la región de América Latina inhiben el desarrollo de los países en el marco de la sociedad del conocimiento a causa de la falta de oportunidades y de inequidad existente en su población, mismas que le limitan la generación del conocimiento científico y tecnológico que les permita verdaderamente resolver sus problemas de contexto (Márquez, 2017).

Cabe señalar que las universidades han estado en un proceso de revisión y mejora de sus procesos académicos con base en alguno de estos modelos desde la década de los años 90, por iniciativa de diferentes organismos nacionales e internacionales preocupados por la calidad educativa (Díaz Barriga, 2019; Gaffigna et al., 2020; Tünnermann, 2008). En este ámbito ha sobresalido el enfoque de competencias que, aunque generó diversos debates en los primeros años del 2000, terminó por ser el planteamiento hegemónico en los sistemas e instituciones educativas, por su énfasis en responder a los requerimientos del mundo organizacional (Díaz Barriga, 2019; Glaesser, 2019). En la actualidad, más que responder a los requerimientos y demandas de las organizaciones, las IES deben formar para superar los problemas del entorno con base en la investigación, la vinculación con las comunidades, y el trabajo colaborativo (GarcíaValdés y Juárez-Hernández, 2019). Por ello, la necesidad de avanzar hacia un nuevo modelo educativo, centrado en el desarrollo social sostenible a partir de la socioformación (García-Rubio et al., 2019; AguilarEsteva et al., 2019).

\section{División del modelo educativo socioformativo}

De acuerdo con los retos de la formación para el desarrollo social sostenible (García-Valdés y JuárezHernández, 2019; United Nations, 2015; UNESCO, 2017), así como los resultados de investigaciones sobre el enfoque socioformativo en los ámbitos de construcción de modelos educativos (Aguilar-Esteva et al., 
2019; Valles-Baca et al., 2019), la gestión curricular (Martínez et al., 2019; Tobón, 2017), la cultura de calidad institucional (Martínez et al., 2017), el desarrollo del talento humano (Tobón y Luna-Nemecio, 2020), la formación integral (Ambrosio, 2018), entre otras aportaciones, el modelo educativo socioformativo se subdivide en los siguientes ejes:

\section{Direccionamiento académico}

Son las orientaciones generales en torno a la formación, la investigación, la gestión y la vinculación con la comunidad que tiene una institución educativa, en donde todos sus miembros asumen sus responsabilidades en sus elementos básicos, aceptando sus respectivas discrepancias (Cebrián et al., 2020; Ambrosio, 2018).

\section{Gestión de la calidad académica}

Se refiere a la implementación de acciones para lograr que los estudiantes se formen de manera integral y pertinente, con la finalidad de contribuir al logro del desarrollo social sostenible, con base en el trabajo de un equipo de actores universitarios por cada programa universitario, departamento o facultad, de manera que se desarrollen los conocimientos, habilidades y actitudes requeridas para afrontar los problemas del entorno (García-Rubio et al., 2019). Implica la conformación de equipos que busquen que el currículo sea pertinente, flexible y acorde con las necesidades de la sociedad (Ilori y Ajagunna, 2020; Martínez et al., 2019; VallesBaca et al., 2019). En este sentido, se vuelve importante que desde la planeación institucional de las comunidades universitarias se establezcan las políticas relacionadas con: proyectos de investigación, evaluación de los aprendizajes, vinculación con la sociedad, incubación de negocios, titulación de los egresados, formación del profesorado, acreditación de la calidad, evaluación y retroalimentación del modelo y programas educativos institucionales, etc. (Martínez et al., 2019; Tobón, 2017).

\section{Formación de los estudiantes}

Es la implementación de acciones concretas con los estudiantes para apoyarlos en su formación integral y la vinculación con la sociedad y la protección del ambiente. Para ello es indispensable llevar a cabo un seguimiento continuo de los estudiantes, con el propósito de diagnosticar y fortalecer su perfil de ingreso, retroalimentar su desempeño de manera continua, implementar proyectos transversales que posibiliten de manera colaborativa el emprendimiento de estrategias que conduzcan al desarrollo social sostenible y al desarrollo de las competencias profesionales de egreso enunciadas en los programas educativos institucionales (Colombo y Alves, 2017; Martínez et al., 2019; Valles-Baca et al., 2019). Cabe señalar que la formación que reciban los estudiantes debe brindarles las herramientas necesarias para mejorar su calidad de vida, así como resolver los problemas que aquejan a la humanidad, tales como: el desempleo, las crisis económicas, el deterioro ambiental, la pérdida de valores, entre otros (García-Rubio et al., 2019).

\section{Gestión del trabajo de los docentes}

Son las acciones orientadas al desarrollo continuo del talento en los docentes para que acompañen a los estudiantes en su formación integral, considerando los retos del contexto e integrando la investigación y la vinculación con la comunidad (Aliaga-Pacora y Luna-Nemecio, 2020; Tobón y Luna-Nemecio, 2020). Esto es importante dentro del modelo educativo socioformativo, debido a que son los docentes los que terminan por guiar la conducción de los proyectos encaminados a desarrollar competencias para la sostenibilidad en los estudiantes (Aliaga-Pacora y Luna-Nemecio, 2020; García-Rubio et al., 2019). Por ello es fundamental que las IES tengan definidas sus políticas de reclutamiento, selección, formación continua, evaluación, promoción y reconocimiento de las prácticas docenes, así como de evaluación de los aprendizajes, con la finalidad de asegurar la formación de emprendedores que contribuyan al desarrollo social sostenible (Tobón, 2017).

\section{Gestión del trabajo de las autoridades}

Es desarrollar el talento de manera continua de los directivos en sus diferentes puestos, con la finalidad de articular la formación integral, la investigación, la vinculación con el contexto y la gestión con base en proyectos colaborativos que tengan impacto en el emprendimiento (Tobón y Luna-Nemecio, 2020). Para ello es importante considerar acciones como el reclutamiento, la selección, la formación, la evaluación, las prácticas directivas, el reconocimiento y la promoción (Tobón, 2017).

\section{Seguimiento y apoyo a los egresados}

Son las diversas acciones que se instrumentan para hacerle un seguimiento a los egresados respecto a la continuación de sus estudios, la vinculación laboral y los procesos de emprendimiento, y con base en esto, 
brindarles acompañamiento mediante actividades de formación continua y extensión (Garzón, 2018). Esto implica la vinculación de los egresados con actividades de la institución, tales como la participación en proyectos de investigación, académicos y culturales.

\section{Servicios de apoyo para la calidad de vida}

La institución ofrece acciones para mejorar la calidad de vida de todos sus actores, y busca la inclusión y la equidad en sus actividades (Garzón, 2018). Esto comprende acciones de bienestar universitario y la eliminación o disminución de barreras para que todos los actores puedan formarse y participar en acciones de investigación, vinculación y gestión (Ambrosio, 2018).

\section{Vinculación del modelo educativo socioformativo}

Un modelo educativo desde la socioformación se vincula con múltiples elementos, porque es transversal y transdisciplinario. Sin embargo, los ejes prioritarios de la vinculación que se deben considerar son los siguientes: epistemología del pensamiento complejo, gestión de la calidad y cambio de la cultura organizacional. A continuación, se explica cada uno de estos tres ejes.

\section{Epistemología del pensamiento complejo}

La epistemología del pensamiento complejo en la versión de Morin (1999) brinda una serie de lineamientos para elaborar, instrumentar y mejorar de manera continua el modelo educativo socioformativo, como los siguientes: 1) articular saberes de varias disciplinas para conceptualizar el desarrollo social sostenible y la formación integral necesaria para lograr este, mediante la multidisciplinariedad, interdisciplinariedad y transdisciplinariedad; 2) integrar los siete saberes necesarios para la educación del futuro dentro del modelo, con el fin de que este aborde al ser humano en su unidad y diferencia, considerando la humanidad en su conjunto; 3) asumir el modelo educativo socioformativo con flexibilidad, buscando el cambio continuo con el fin de integrar los retos progresivos de la comunidad y la sociedad en su conjunto; y 4) integrar la antropoética como un camino que deben seguir todos los actores universitarios en sus acciones.

\section{Gestión de la calidad académica}

En todo modelo educativo basado en la socioformación se tienen en cuenta los criterios de calidad y/o acreditación establecidos por los organismos nacionales e internacionales, de tal manera que se cumpla con los retos de desarrollo de la comunidad, pero también con los criterios básicos establecidos por las autoridades u organismos competentes de evaluar la calidad de los estudios universitarios (Martínez et al., 2017). Sin embargo, a diferencia de otros enfoques para la educación superior, en la socioformación la gestión de la calidad es parte del trabajo de todos los actores, incluyendo a los alumnos, ya que se busca mejorar de manera permanente las acciones de formación para responder a la resolución de los problemas que afectan el desarrollo social sostenible (García-Rubio et al., 2019; Martínez et al., 2017).

\section{Cambio de la cultura organizacional}

Las IES son organizaciones en las cuales se tiene una cultura de trabajo que en muchos casos es implícita, y esta cultura orienta las actuaciones de los diferentes actores respecto al proceso de formación, evaluación del aprendizaje, investigación, vinculación con la comunidad y gestión (Bauer et al., 2020; Ortiz-Colón et al., 2017). Para poder generar cambios perdurables, es esencial comprender la cultura que prevalece, reconocer sus logros pero también identificar sus áreas de oportunidad, y con base en ello poner en funcionamiento mejoras y cambios a través del trabajo participativo de la comunidad académica, mediante el seguimiento, los planes de acción a largo plazo, la introducción de cambios paulatinos, la promoción de los logros y la valoración del trabajo de los actores involucrados (Ambrosio, 2018; Bauer et al., 2020; OrtizColón et al., 2017). Es preciso reconocer la resistencia al cambio y cómo afrontarla, empleando diversas estrategias construidas en el ámbito de las organizaciones sociales, empresariales, culturales, científicas y tecnológicas.

\section{Metodología de construcción del modelo educativo socioformativo}

En la literatura hay pocas referencias sobre el proceso de construcción de un modelo educativo. Desde el enfoque educativo de la socioformación, los pasos para su construcción en las IES son (Aguilar-Esteva et al., 2019; Tobón, 2017; Valles-Baca et al., 2019):

\section{Establecimiento del trabajo colaborativo}

Consiste en conformar un proceso de trabajo colaborativo con el propósito de crear, reconstruir o mejorar el modelo educativo que actualmente posee la institución, con base en un diagnóstico y considerando los retos 
en la formación, la investigación, la vinculación y la gestión, y teniendo también en cuenta los requerimientos legales y de acreditación de los programas. Para ello, el equipo debe acordar, junto con las autoridades, un plan de acción con metas, productos a entregar, actividades y fechas tentativas, que posibiliten evaluar el trabajo. Se recomienda que el equipo de trabajo esté conformado por actores comprometidos y motivados con este proceso, como autoridades, docentes, estudiantes y egresados. Además, es necesario que el equipo se capacite en qué es un modelo educativo socioformativo, cómo se construye y cuál debe ser su orientación.

\section{Diagnóstico de necesidades}

En esta fase se lleva a cabo un análisis de las necesidades del ámbito internacional, nacional, regional e institucional, con la finalidad de que el futuro modelo educativo socioformativo oriente a la institución del nivel superior a realizar una gestión curricular acorde con sus retos y problemas de contexto más imperantes. Esto implica, por ejemplo, determinar cómo son las prácticas docentes y directivas, en qué niveles se están desarrollando las competencias genéricas, cuál es el grado de emprendimiento en los alumnos, qué impacto se tiene en investigación, cómo se está apoyando a la comunidad en la resolución de sus problemas y cómo son las tasas de deserción y titulación. Un ejemplo de diagnóstico institucional para elaborar el modelo educativo socioformativo se encuentra en Valles-Baca et al. (2019).

\section{Establecimiento de los componentes mínimos a seguir}

El equipo de trabajo en conjunto con diversos miembros de la comunidad educativa (profesores, directivos, estudiantes, personal administrativo, padres de familia y miembros de la sociedad) debe establecer: 1) el tipo de persona que se pretende formar; 2) los enfoques educativos que orientarán el trabajo en la institución; 3) el tipo de sociedad que se busca construir; 4) el proceso curricular a seguir y la gestión de la calidad académica; 5) los lineamientos para la docencia, la formación y la evaluación; 6) los principios filosóficos por considerar; 7) los ejes de la investigación y del emprendimiento; y 8) la orientación en torno a la vinculación con la comunidad.

\section{Evaluación del modelo educativo socioformativo}

Concluida la elaboración del modelo educativo socioformativo, este debe de ser enviado a pares académicos externos, con la finalidad de que el documento sea evaluado y reciba recomendaciones que permitan mejorar. Se recomienda también socializar el modelo educativo socioformativo con la comunidad universitaria para recibir sugerencias que ayuden a fortalecer los ejes de este, sin embargo, para poder desarrollar dicho proceso, es de vital importancia que la misma institución elabore un instrumento de evaluación de su modelo educativo.

\section{Socialización del modelo educativo socioformativo}

Atendidas las observaciones emitidas por los pares académicos externos y la misma comunidad universitaria, se procede a dar a conocer en diversos medios el modelo educativo institucional. Es importante compartir el modelo educativo socioformativo con las organizaciones sociales, empresariales, profesionales y de investigación vinculadas con la institución.

\section{Formación de la comunidad educativa}

En esta fase se capacita a la comunidad educativa en la aplicación del modelo educativo socioformativo, a través de diferentes acciones. No es suficiente dar a conocer el modelo, es preciso un proceso de formación en torno a este. Para ello, se sugiere un curso de formación en línea continuo para todos los actores, con algunos talleres presenciales cada semestre. El propósito debe ser que el modelo se aplique en los procesos de formación, investigación, vinculación y gestión.

\section{Implementación y evaluación del modelo educativo socioformativo}

Aplicado el modelo educativo socioformativo en los procesos de formación, investigación, vinculación y gestión institucional, y después de un periodo de tiempo, es necesario evaluar el modelo educativo, con la finalidad de revisar su relación con las necesidades de contexto y con los programas educativos que la institución educativa oferta, así como el grado de asimilación por parte de la comunidad educativa.

\section{Ejemplificación del modelo educativo}

En la Tabla 2 se presenta un ejemplo de elaboración de un modelo educativo para la formación universitaria acorde con los retos del desarrollo social sostenible aplicando la socioformación. 
Tabla 2: Ejemplo de elaboración de un modelo educativo desde la socioformación

Institución: Universidad de las Humanidades.

Localización: Cuernavaca, Morelos, México.

Tipo de institución: Pública.

Número de campus: 2 .

Número de programas educativos que se ofertan: 10 licenciaturas y 4 maestrías.

Número de estudiantes inscritos: 1112 en licenciatura y 82 en posgrado.

Número de profesores: 58 profesores de tiempo completo y 42 de asignatura.

Contexto de la institución: La Universidad de las Humanidades es una institución pública que en la actualidad no cuenta con un modelo educativo, el cual se considera necesario, ya que ofrece programas educativos de licenciatura y de posgrado en sus dos campus, además de desarrollar proyectos de investigación en el área de las humanidades y las ciencias sociales.

1. Establecimiento $\quad$ En la Universidad de las Humanidades, se conformó un equipo de trabajo de 6 personas para liderar 1. Establecimiento colaborativo la construcción del modelo educativo con enfoque socioformativo de la institución. Para ello, establecieron un plan de trabajo con metas por alcanzar, actividades a realizar y fechas de reunión con otros actores de la comunidad educativa, tales como directivos, profesores, personal administrativo, estudiantes, padres de familia y representantes de organizaciones sociales. El plan de trabajo se hizo para ser desarrollado durante un semestre, con el fin de dedicar otro semestre a la capacitación de todos los actores en este proceso.

2. Diagnóstico de $\quad$ El equipo líder del proyecto realizó un diagnóstico amplio y sistemático de las necesidades, el cual necesidades. $\quad$ reflejó lo siguiente:

El contexto internacional requiere que las IES establezcan procesos de gestión curricular tendientes a formar personas integrales que contribuyan al desarrollo social sostenible, con una perspectiva global. En lo nacional se estableció como política educativa la necesidad de que los programas educativos se orienten a la atención de los problemas de contexto, aborden los cambios en el empleo por la emergencia de la tecnología y se acrediten ante un organismo externo a la institución

Regionalmente impera el desempleo en egresados universitarios, implicando el $80 \%$ de las carreras que ofrece la institución.

A nivel institucional, se encontró: 1) prácticas pedagógicas enfocadas en contenidos en más del $75 \%$ de las clases observadas; 2) prima la evaluación orientada a las calificaciones y únicamente es práctica la evaluación formativa en un $10 \%$ de las clases observadas; 3) los procesos de emprendimiento son escasos, pues solamente hay registro de un $5 \%$ de alumnos con algún proyecto; 4) el número de docentes que aborda la investigación es inferior al 8\%; 5) la deserción es del 54\%; 6) la tasa de titulación en el tiempo establecido es del $30 \%$ y 7 ) más de un $35 \%$ de alumnos no tiene adecuados niveles de lectura comprensiva, redacción argumentativa y trabajo colaborativo.

3. Establecimiento de los El equipo líder acuerda con las autoridades y diversos actores de la Universidad los siguientes ejes esenciales del modelo educativo socioformativo, con base en varias reuniones y talleres de reflexión:

componentes mínimos a seguir. Se adopta como enfoque educativo la socioformación para orientar los diversos procesos institucionales, tomando en cuenta los principios del pensamiento complejo de Morin.

La formación, la investigación, la vinculación con la sociedad y la gestión se orientarán al desarrollo social sostenible por medio del emprendimiento. Esto implica la implementación de la resolución de problemas como metodología clave de formación de todos los estudiantes desde el inicio de sus carreras y en todo el microcurrículo. Se propenderá por abordar problemas de alto impacto que afecten a la comunidad y a las organizaciones.

Las carreras se orientarán a la resolución de retos en el contexto, y el microcurrículo tendrá como eje esencial los proyectos formativos. Se tendrán en cuenta los cambios en las profesiones y el empleo, como también los criterios de acreditación desde el inicio. Se espera que en cinco años, el $80 \%$ de los programas tengan acreditación de calidad reconocida por un organismo acreditador.

El docente debe de ser un mediador para el emprendimiento, buscando que los alumnos aborden retos del entorno con base en la búsqueda, apropiación y aplicación del saber científico y tecnológico. Para ello, se aplicará la evaluación continua que lleve a la formación y a la mejora, por medio de productos pertinentes en el contexto.

En los proyectos formativos de las carreras y posgrados se integrarán la investigación y la vinculación como ejes transversales, a partir de líneas de investigación definidas por las facultades.

4. Evaluación del modelo educativo socioformativo.

Concluido el proceso de construcción del modelo educativo socioformativo, el documento es enviado a pares académicos externos a la institución, quienes emiten su dictamen de aprobación, así como sugerencias de mejora. Esto mismo se hará con la comunidad educativa.

5. Socialización del modelo educativo socioformativo.

Atendidas las observaciones emitidas por los pares académicos, el modelo educativo socioformativo de la institución es socializado a través de diversos medios, tales como: eventos de presentación ante la comunidad educativa, cuadernillos impresos y electrónicos, folletos, página web de la institución, redes sociales, entre otros.

\begin{tabular}{ll}
$\begin{array}{l}\text { 6. Formación de la } \\
\text { comunidad } \\
\text { educativa. }\end{array}$ & $\begin{array}{l}\text { La institución establece diversos tipos de cursos y en diversas modalidades de aprendizaje } \\
\text { (presencial, semipresencial o a distancia), con la finalidad de que la comunidad educativa se forme } \\
\text { acorde a las características del modelo educativo socioformativo y los retos del desarrollo social } \\
\text { sostenible. }\end{array}$ \\
\hline $\begin{array}{l}\text { 7. Implementación } \\
\text { y evaluación del } \\
\text { modelo educativo } \\
\text { socioformativo }\end{array}$ & $\begin{array}{l}\text { La institución procede a aplicar el modelo educativo socioformativo en los ámbitos de formación, } \\
\text { investigación, vinculación y gestión institucional, sin embargo, acuerda que en un periodo de } 5 \text { años } \\
\text { llevará a cabo la evaluación externa e interna del modelo educativo, con la finalidad de revisar la } \\
\text { relación que guardan los programas educativos con la filosofía institucional, así como verificar la } \\
\text { pertinencia de la universidad con las necesidades que presenta la sociedad. }\end{array}$ \\
\hline
\end{tabular}




\section{DISCUSIÓN}

De acuerdo con los resultados del estudio efectuado, la formación universitaria es fundamental para el logro del desarrollo social sostenible. Esto implica que las IES establezcan una visión compartida en torno a los problemas que aquejan a la comunidad, con la finalidad de establecer acciones a través de la docencia, la colaboración, la investigación y vinculación con los diversos sectores de la sociedad (Juárez-Hernández et. al., 2019), y así poder desarrollar en los estudiantes y demás miembros de las comunidades educativas, las competencias requeridas para la sostenibilidad (Aliaga-Pacora y Luna-Nemecio, 2020; Colombo y Alves, 2017; García-Rubio et al., 2019).

Si bien hay un aumento considerable del número de IES que llevan a cabo la creación o mejoramiento de sus modelos educativos con base en las competencias $u$ otros enfoques educativos que han propuesto establecer al estudiante como el centro del proceso de aprendizaje (Barbosa y Amariles, 2019; Gaffigna et al., 2020; Martínez et al., 2017; Solís et al., 2015), la realidad es que estos no han sido comprendidos ni asimilados por las instituciones del nivel superior (Guzmán, 2017; Solís et al., 2015), ni tampoco han logrado direccionar las prácticas educativas a la solución de los problemas que aquejan a la humanidad, como el desempleo, la desigualdad social, los problemas de salud, el deterioro ambiental, entre otros. En cierta forma, esto obedece a causa de que los modelos educativos implementado por gran número de IES mantienen en su esencia prácticas educativas tradicionales como la enseñanza contenidos, la evaluación por medio de pruebas estandarizadas, estructuras curriculares por asignaturas aisladas, entre otras prácticas que poco o nulo impacto tienen en la mejora de la sociedad (Díaz Barriga, 2019; Martínez et al., 2019). Por ello, la importancia de hacer énfasis en la propuesta del modelo educativo socioformativo como una alternativa de formación integral de los actores de las comunidades universitarias con una visión compartida de los problemas que aquejan a las IES y a la sociedad en general (Aguilar-Esteva et al., 2019).

Desde la socioformación, se propone un modelo educativo que desde su construcción o rediseño cuente con los aportes y experiencias de cada uno de los actores que convergen dentro y fuera de una institución educativa, con la finalidad de centrar las prácticas formativas en la resolución de aquellos problemas que más aquejan a la sociedad, por medio de la colaboración, el pensamiento complejo, la mejora y evaluación continua del desempeño, la flexibilidad curricular que permita la adaptación a los cambios, así como la responsabilidad de los actos que se efectúan de manera individual y colectiva (Ambrosio, 2018; Tobón, 2017; Martínez et al., 2019). Cabe señalar que una institución educativa del nivel superior que aplique el modelo educativo socioformativo comprende que su fin primordial es la formación integral del estudiante y demás miembros de la comunidad educativa, por lo que considera al desarrollo social sostenible como una oportunidad para cambiar la cultura institucional, desarrollar el talento humano de los diversos actores educativos y proponer alternativas que nos lleven a solucionar problemas reales mediante el emprendimiento de proyectos (García-Rubio et al., 2019; Valles-Baca et al., 2019), articulando la docencia, la investigación y la vinculación con los diversos sectores sociales (Juárez-Hernández et al., 2019), y de esta forma, mejorar la calidad de vida de las personas y de las comunidades.

\section{CONCLUSIONES}

A partir del análisis llevado a cabo, se concluye lo siguiente:

1.- La sociedad de nuestros tiempos presenta varios problemas que ponen en riesgo el desarrollo de la humanidad, tales como la pobreza, el desempleo, la violencia, los problemas de salud, la contaminación del ambiente, etc. En este sentido, los modelos educativos fundamentados en diversos enfoques (como las competencias o el constructivismo, por mencionar algunos) que han implementado las IES no han orientado de manera puntual las prácticas educativas hacia la resolución de los problemas que más afectan a la sociedad. Por ello, se requiere la incorporación del desarrollo social sostenible y, por ende, enfoques pedagógicos más pertinentes a los retos actuales, como es la socioformación, una propuesta de origen latinoamericano que se centra en formar con base en estrategias que posibiliten afrontar los retos y mejorar las condiciones de vida y el cuidado del ambiente.

2.- El modelo educativo socioformativo promueve realizar el diagnóstico de las necesidades instituciones y comunitarias en general, con el propósito de conducir la formación integral de los estudiantes y demás actores universitarios por medio del emprendimiento de proyectos transversales que contribuyan al desarrollo social sostenible, integrando la docencia, la gestión, la investigación y vinculación con los diversos sectores de la sociedad. Entre las principales características del enfoque educativo de la socioformación se encuentran la resolución de problemas de contexto, el trabajo colaborativo, la evaluación orientada al desempeño, el establecimiento de un proyecto ético de vida que orienta la forma de actuar de las personas, la visión de las situaciones de vida con pensamiento complejo, así como la flexibilidad y mejora continua del currículo. 
3.-Para construir o mejorar un modelo educativo desde el enfoque de la socioformación, es necesario contar con la participación de diversos actores educativos (docentes, directivos, estudiante, egresados, expertos y organizaciones sociales y empresariales), así como conformar un equipo líder con personas comprometidas con este proceso, que no solamente participen en su construcción o mejora, sino también en su puesta en práctica, seguimiento y evaluación. El modelo educativo socioformativo promueve una visión compartida que se aplica en todos los ámbitos institucionales, promoviendo la autoevaluación y mejora continua de las prácticas educativas para que estas no queden en el discurso, tal y como ha ocurrido en la implementación de modelos educativos fundamentados en otros enfoques que poco o nada contribuyen a la formación universitaria para el desarrollo social sostenible.

\section{REFERENCIAS}

Aguilar-Esteva, V., Tobón-Tobón, S., y Juárez-Hernández, L.G., Construcción y validación de instrumento para evaluar el avance del enfoque socioformativo y adopción de nuevos modelos educativos en el nivel superior en México, Espacios, 40(31), 1-14 (2019).

Aliaga-Pacora, A.A., y Luna-Nemecio, J., La construcción de competencias investigativas del docente de posgrado para lograr el desarrollo social sostenible, Espacios, 41(20), 1-12 (2020).

Ambrosio, R., La socioformación: un enfoque de cambio educativo, https://doi.org/10.35362/rie7612955, Revista Iberoamericana de Educación, 76(1), 57-82 (2018).

Andriamihaja, O.R., Metz, F., y otros tres autores, Identifying agents of change for sustainable land governance, https://doi.org/10.1016/j.landusepol.2020.104882, Land Use Policy, 100, 1-12 (2021).

Barbosa, S.H., y Amariles, M.L., Learning styles and the use of ICT in university students within a competency-based training model, https://doi.org/10.7821/naer.2019.1.296, Journal of New Approaches in Educational Research, 8(1), 1-6 (2019).

Bauer, M., Niedlich, S., y otros tres autores, Interdependencies of culture and functions of sustainability governance at higher education institutions, https://doi.org/10.3390/su12072780, Sustainability, 12(7), 1-21 (2020).

Cebrián, G., Junyent, M., y Mula, I., Competencies in education for sustainable Development: emerging teaching and research developments, https://doi.org/10.3390/su12020579, Sustainability, 12(2), 1-9 (2020).

Colombo, C.R., y Alves, A.C., Sustainability in engineering programs in portuguese public university, https://doi.org/10.1590/0103-6513.221416, Prod., 27(spe), 1-16 (2017).

Delors, J., La educación encierra un tesoro. Informe a la UNESCO de la Comisión Internacional sobre Educación para el Siglo XXI, UNESCO, París, Francia (1996).

Díaz Barriga, F., Evaluación de competencias en educación superior: experiencias en el contexto mexicano, https://doi.org/10.15366/riee2019.12.2.003, Revista Iberoamericana de Evaluación Educativa, 12(2), 49-66 (2019).

Echeverría, B., y Martínez, P., Revolución 4.0, competencias, educación y orientación, https://doi.org/10.19083/ridu.2018.831, Rev. Digit. Invest. Docencia Univ., 12(2), 4-34 (2018).

Eryaman, M.Y., Discipline-based curriculum; In Encyclopedia of curriculum estudies by C. Kridel, pp. 294, SAGE Publications, Thousand Oaks, CA (2010).

Fresán, M., Moreno, T., y otros tres editores, Modelos educativos para el siglo XXI. Aproximaciones sucesivas, UAM, Unidad Cuajimalpa, Ciudad de México (2017).

Gaffigna, A. M., Ghilardi, L. M., y Dávila, M. A., Analysis of curriculum processes for the development of competencies in engineering education, https://doi.org/10.18543/tjhe-7(2)-2020pp25-42, Tuning Journal for Higher Education, 7(2), 25-42 (2020).

García-Rubio, M.P., Becerra-Sarmiento, M.F., y otros tres autores, El modelo educativo socioformaivo: impacto sobre los niveles de empleo, Revista Ibérica de Sistemas e Tecnologias de Informação, E21, 208-214 (2019).

García-Valdés, M.G., y Juárez-Hernández, L.G., Diseño y validación de un instrumento para evaluar la formación en sostenibilidad en estudiantes de educación superior, https://doi.org/10.33010/ie_rie_rediech.v10i19.501, IE Rev. investig. educ. REDIECH, 10(19), 37-54 (2019).

Garzón, A., Modelo para el Seguimiento y Acompañamiento a Graduados (SAG), una visión holística de la gestión de la calidad de la educación superior, https://doi.org/10.18800/educacion.201801.011, Educación, 27(52), 201-2018 (2018).

Glaesser, J., Competence in educational theory and practice: a critical discussion, https://doi.org/10.1080/03054985.2018.1493987, Oxford Review of Education, 45(1), 70-85 (2019).

Guzmán, F., Problemática general de la educación por competencias, https://doi.org/10.35362/rie740610, Revista Iberoamericana de Educación, 74, 107-120 (2017).

Heyneman, S.P., y Lee, B., International organizations and the future of education assistance, https://doi.org/10.1016/j.jjedudev.2015.11.009, International Journal of Educational Development, 48, 9-22 (2016). 
Ilori, M.O., y Ajagunna, I., Re-imagining the future of education in the era of the fourth industrial revolution, https://doi.org/10.1108/WHATT-10-2019-0066, Worldwide Hospitality and Tourism Themes, 12(1), 3-12 (2020).

Jim, Y.C., y Shen, J.P., Higher education for sustainable development: a systematic review, https://doi.org/10.1108/lJSHE-01-2015-0004, International Journal of Sustainability in Higher Education, 17(5), 633-651 (2016).

Juárez-Hernández, L.G., Tobón, S., y otros tres autores, Desarrollo sostenible: educación y sociedad, M+A, Revista Electrónica de Medio Ambiente de la UCM, 20(1), 54-72 (2019).

Kangas, R., y Aarrevaara, T., Higher education institutions as Knowledge brokers is smart specialisation, https://doi.org/10.3390/su12073044, Sustainability, 12(7), 4-15 (2020).

Márquez, A., Educación y desarrollo en la sociedad del conocimiento, Perfiles Educativos, 29(158), 3-17 (2017).

Martínez, J.E., Tobón, S., y López, E., Currículo: un analisis desde un enfoque socioformativo, https://doi.org/10.33010/ie_rie_rediech.v10i18.200, IE Rev. investig. educ. REDIECH, 10(18), 43-63 (2019).

Martínez, J.E., Tobón, S., y Romero, A., Problemáticas relacionadas con la acreditación de la calidad de la educación superior en América Latina, Innovación Educativa, 17(73), 79-96 (2017).

Morin, E., Seven complex lessons in education for the future, UNESCO, Paris, France (1999).

OECD, OECD Skills Strategy Diagnostic Report: Mexico 2017, OECD Publishing, París, Francia (2017).

Ortega, M.F., Hernández, J.S., y Tobón, S., Análisis documental de la gestión del conocimiento mediante la cartografía conceptual, Ra Ximhai, 11(4), 141-160 (2015).

Ortiz-Colón, A. M., Ovelar, L.A., y otros dos autores, Organizational culture from the teaching perspective in higher education, Espacios, 38(60), 1-12 (2017).

Peña, T., y Pirela, J., La complejidad del análisis documental, https://doi.org/10.34096/ics.i16.869, Información, Cultura y Sociedad, 16, 55-81 (2007).

Ricaurte, P., Pedagogies for the open knowledge society, https://doi.org/10.1186/s41239-016-0033-y, Int. J. Educ. Technol. Hig. Educ., 13, 1-10 (2016).

Rolón, V., Enfoques curriculares en la educación superior, Academo. Revista de Investigación en Ciencias Sociales y Humanidades, 3(1), 1-10 (2016.

Schwab, K. The fourth industrial revolution, World Economic Forum, Geneva, Switzerland (2016).

Solís, L.E, Baeza, J.R., y otros dos autores, Estudio comparativo de dos modelos educativos basado en los resultados del rendimiento académico de los alumnos de licenciatura en ingeniería, https://dx.doi.org/10.4067/S071850062015000300006, Form. Univ., 8(3), 47-56 (2015).

Tobón, S., Formación integral y competencias. Pensamiento complejo, currículo, didáctica y evaluación, ECOE Ediciones, Bogotá, Colombia (2013).

Tobón, S., Curricular Management and socioform0ative approach, Kresearch Corporation, Lake Mary, USA (2017).

Tobón, S., Juárez-Hernández, L.G., y otros dos autores, Assessing school principal leadership practices. Validity and reliability of a rubric, https://doi.org/10.5944/educXX1.23894, Educación XXI, 23(2), 187-210 (2020).

Tobón, S., y Luna-Nemecio, J., Proposal for a new talent concept based on socioformation, https://doi.org/10.1080/00131857.2020.1725885, Educational Philosophy and Theory, 1-13 (2020).

Tünnermann, C., Modelos eductaivos y académicos, Editorial Hispamer, Nicaragua (2008).

UNESCO, Education for sustainable development goals: Learning objectives, Publishing UNESCO, Paris, France (2017).

United Nations, Transforming our world: The 2030 agenda for sustainable development, United Nations, New York, EE. U.U., (2015).

Valles-Baca, H.G., Parra-Acosta, H.S. y otros cinco autores, El modelo educativo y sus implicaciones en la formación de estudiantes de licenciatura y posgrado para la sociedad del conocimiento, Universidad Autónoma de Chihuahua, Chihuahua (2019). 